\title{
Evolución del mundo material en los cuidados de enfermería: siglos XVIII al XX
}

Evolution of the material world in nursing care: XVIII to XX centuries

\section{Evolução do mundo material no cuidado de enfermagem: XVIII a XX}

\author{
Sonia Sánchez Aragó \\ Graduada en enfermería \\ Cómo citar este artículo en edición digital: Sánchez-Aragó, S. (2017). Evolución del mundo material en los cuidados de \\ enfermería: siglos XVIII al XX. Cultura de los Cuidados (Edición digital), 21(49). \\ Recuperado de http://dx.doi.org/10.14198/cuid.2017.49.09 \\ Correspondencia: Remitirse al correo electrónico. \\ Correo electrónico: sonits_@hotmail.com \\ Recibido: 09/02/2017; Aceptado: 03/06/2017
}

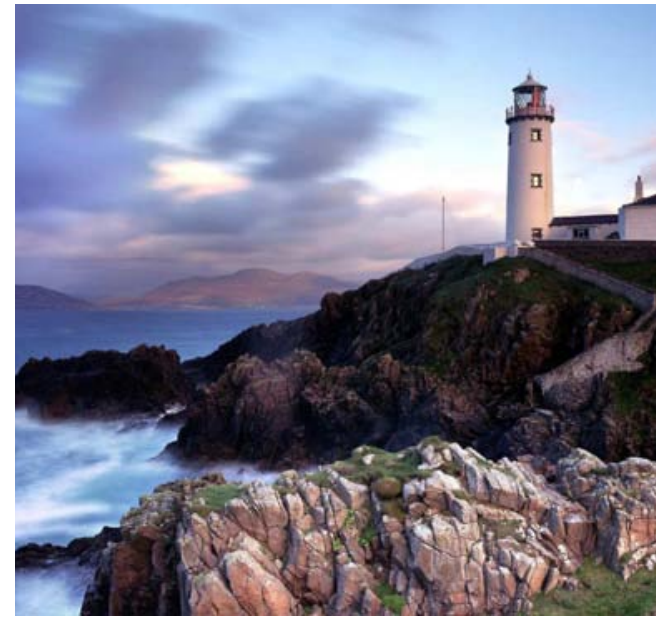

\section{ABSTRACT}

Throughout the evolution of the nursing profession, have carried out various scenarios care and have used various materials to provide care. The material world of nursing care consists of a wide variety of instruments that support such care, facilitating the work of nursing. It is therefore important to know the elements that our predecessors used in nursing practice and its most important features

Objective: It aims to analyze the situation of the material world throughout history in- cluding nursing between the eighteenth and twentieth centuries, in order to gather information and present an updated version of what instruments vision were used and how it is carried out carefully.

Methods: It has done a literature review in order to find and use information available about the material world throughout the history of nursing. It has also been carried out the analysis of various manual to understand the care provided in those centuries

Results: technological, scientific and social challenges throughout the seventeenth movements, and especially nineteenth and twentieth centuries, there have been several changes in the material world and the stage of care in the nursing profession

Conclusions: he analysis of media and objects used in the care, allows you to draw an overview of the evolving role of nurses. The material world of care has been built and enriched by the various figures who have dedicated themselves to caring and objects that have been part of that care

Keywords: history of nursing, techonology, cares, health, nursing. 


\section{RESUMEN}

A lo largo de la evolución de la profesión enfermera, se han llevado a cabo cuidados en diversos escenarios y se han utilizado diversos materiales para proporcionar cuidados. El mundo material de los cuidados de enfermería está formado por una amplia variedad de instrumentos que dan soporte a esos cuidados, facilitando la labor de enfermería. Por ello, es importante conocer los elementos que utilizaron nuestros predecesores en la práctica enfermera y sus características más relevantes.

Objetivos: En este trabajo se pretende analizar la situación del mundo material a lo largo de la historia de enfermería comprendida entre los siglos XVIII y XX, con el fin de recopilar información y exponer una visión actualizada de qué instrumentos se utilizaban y cómo se llevaba a cabo el cuidado.

Metodología: se ha realizado una revisión bibliográfica con el fin de encontrar y utilizar información disponible acerca del mundo material a lo largo de la historia de enfermería. También se ha procedido al análisis de diversos manuales para entender el cuidado proporcionado en esos siglos

Resultados principales: los movimientos tecnológicos, científicos y sociales a los largo de los siglos XVII, y sobre todo XIX y XX, han producido diversas modificaciones en el mundo material y en el escenario de cuidados en la profesión enfermera

Conclusión principal: El análisis de los medios y objetos utilizados en el cuidado, permite trazar una visión general acerca de la evolución del rol de los profesionales de enfermería. El mundo material de los cuidados se ha construido y enriquecido gracias a las diferentes figuras que se han dedicado a cuidar y a los objetos que han formado parte de ese cuidado
Palabras clave: historia de enfermería, tecnología, cuidados, salud, enfermería.

\section{RESUMO}

Introdução: Ao longo da evolução da profissão de enfermagem, levaram a cabo vários cenários de cuidado e ter usado vários materiais para prestar cuidados. $\mathrm{O}$ mundo material dos cuidados de enfermagem é composto por uma grande variedade de instrumentos que suportam tais cuidados, facilitando o trabalho de enfermagem. Por isso, é importante conhecer os elementos que nossos antecessores utilizados na prática de enfermagem e suas características mais importantes.

Objetivos: Este trabalho tem como objetivo analisar a situação do mundo material em toda a história da enfermagem entre os séculos XVIII e XX, a fim de recolher informações e apresentar uma versão atualizada do que a visão instrumentos foram utilizados e como eles carregavam cuidados fora.

Metodologia: Foi realizada uma revisão da literatura a fim de encontrar e utilizar a informação disponível sobre o mundo material em toda a história da enfermagem. Também foi realizada a análise de vários manual para compreender os cuidados prestados nesses séculos.

Principais resultados: movimentos tecnológicos, desafios científicos e sociais em todo o XVII e séculos, especialmente XIX e XX, houve várias mudanças no mundo material e o estágio de cuidados na profissão de enfermagem.

A principal conclusão: A análise dos meios de comunicação e objetos usados no cuidado, permite desenhar um panorama da evolução do papel da enfermagem. O mundo material dos cuidados foi construída e enriquecida pelas várias figuras que se dedicaram a cuidar e objetos que fizeram parte desse cuidado. 
Palavras-chave: história da enfermagem, tecnologia, cuidado, saúde, enfermagem.

\section{INTRODUCCIÓN}

El cuidado como objeto de estudio de enfermería, se ha ido consolidando en las distintas épocas históricas a través de la evolución de los roles que ha cumplido enfermería, junto con el desarrollo científico, el saber filosófico y el posterior desarrollo que ha significado para nuestra disciplina (García, 2013).

Si nos remontamos al siglo XVIII podemos ver que es cuando se expande la necesidad de tratamiento sanitario, pero el mismo tiene un fortísimo déficit de elementos (Martinez,2011).

Así, hasta la aparición de la enfermería como ciencia a mediados del siglo pasado, la profesión de enfermería se basaba en reglas, principios y tradiciones transmitidas a través del aprendizaje y en la sabiduría acumulada a lo largo de los años de experiencia; es decir tenía un sentido vocacional (Sosa, 2010).

Es en el año 1857 la Ley de Bases para la Instrucción Pública, conocida como Ley Moyano por el nombre del ministro que la auspició, estableció la creación de los denominados Practicantes, que agrupaban a los anteriores, más Callistas, Dentistas (hasta 1877) y Asistentes a partos ( Iglesias, 2009). Sin embargo es necesario esperar hasta la mitad del siglo XX, para poder encontrar nuevamente un desarrollo e impulso teórico y científico en los cuidados de enfermería (Bernabéu, 1989). En esta evolución, "practicantes” y "matronas” formarán sus respectivas asociaciones, y actuarán en los hospitales y en la incipiente sanidad pública (Siles,2010).

Posteriormente, de acuerdo con las normativas establecidas en Francia y otros países algunos años atrás, en 1915 se creó en España el Título de Enfermería; los enfermeros/as agrupaban a los Practicantes (hombres), las Enfermeras (mujeres) y a las Matronas. Sin embargo, durando la Guerra Civil Española, la formación de practicantes, matronas y enfermeras quedó paralizada (Ávila,2010). Fue en 1953, dado que en la práctica no se había desarrollado la normativa, cuando se produce la unificación de estos tres profesionales con el nacimiento de los ATS (Ayudantes Técnicos Sanitarios) (Arrendondo y Siles, 2005).

Finalmente, a partir de la primera mitad de los años ochenta la integración definitiva en la Universidad convertirá a los antiguos ATS en Diplomados Universitarios en Enfermería, que en fechas muy recientes, con la aplicación en España de la Reforma del Espacio Europeo de la Educación Superior, ha conducido al Grado en Enfermería (Campos, 2007).

Surge así una revalorización de la persona como sujeto de los cuidados enfermeros, entendida como ser multidimensional, que presenta aspectos tanto físicos, como mentales y sociales. Se abre el campo, en el que la disciplina enfermera puede aportar al conjunto de la ciencia, en toda su complejidad ontológica el cuidado humano, que involucra al " ser cuidador", al "ser cuidado" y al "proceso" (García, 2013).

A lo largo de la evolución de la profesión enfermera, se han llevado a cabo cuidados en diversos escenarios y se han utilizado diversos materiales para proporcionar cuidados. El mundo material de los cuidados de enfermería está formado por una amplia variedad de instrumentos que dan soporte a los cuidados, facilitando la labor de enfermería. Por ello, es importante conocer los elementos que utilizaron nuestros predecesores en la práctica enfermera y sus características más relevantes. En 
este trabajo, se pretende analizar la situación del mundo material a lo largo de la historia de enfermería comprendida entre los siglos XVIII y XX, con el fin de recopilar información y exponer una visión actualizada de qué instrumentos se utilizaban y cómo se llevaba a cabo el cuidado (García, 2013).

\section{METODOLOGÍA}

Se trata de un estudio histórico que pretende relatar cómo se llevaba a cabo el cuidado, cómo eran los espacios que se utilizaban y cómo eran los materiales de los que se disponía para proporcionar cuidados. Para ello, se ha realizado una revisión bibliográfica con el fin de encontrar y utilizar información disponible acerca del mundo material a lo largo de la historia de enfermería. Se ha buscado información en diversas bases de datos tales como Cuiden, Dialnet, Pubmed. También se ha procedido al análisis de diversos manuales para entender el cuidado proporcionado en esos siglos, disponibles de forma gratuita en la página web del Colegio Oficial de Enfermería de Madrid. Entre los manuales analizados se encuentra: Manual de la enfermera (1936), Manual de enseñanza de enfermeras (1917), Manual de flebotomianos o sangradores (1846), Manual para el uso del practicante (1866) y manual del practicante y del interno de hospital (1901).

\section{RESULTADOS}

El siglo XVIII es conocido como Siglo de las Luces debido a que surge un movimiento intelectual conocido como Ilustración. En esta época, las actividades caritativas se asumieron por distintas sectas religiosas o delgadas a las autoridades seglares. En España e Italia, países católicos, las pugnas religiosas no tuvieron efecto directo sobre los hospitales (Arredon- do González, De la Cuesta, Ávila Olivares, 2015).

Existía un grave problema de mortalidad infantil, ya que el cuidado prenatal era deficiente o nulo y se proporcionaba a los bebes una alimentación inadecuada debido a la ignorancia y a la pobreza, además estaba muy presente la fiebre puerperal (Campos, 2007).

A esto hay que unir, los brotes devastadores de epidemias, ya que el estilo de vida urbano empezaba a favorecer estas enfermedades. Como regla general, la higiene era deficiente y existía carencia de instalaciones sanitarias. Además, la depuración de las aguas residuales era inadecuada, y no había leyes de salud pública ( Santo-Tomás,1994)

Los hospitales de las ciudades no poseían ninguna decoración y consistían en pequeñas habitaciones oscuras con ventanas pequeñas por las que apenas entraba el sol. Normalmente, en las salas lúgubres se amontonaban cincuenta o cien pacientes Las sangrías y las purgas eran los tratamientos habituales cuando se presentaba cualquier dolencia. En las salas, las camas estaban muy próximas entre sí y la limpieza se hacía casi imposible. Por otro lado, el aseo de los enfermos no era un procedimiento habitual. (Herrera, 2003). A la hora de dirigir los hospitales, se nombraba a hombres civiles y las seglares (así era como se llamaba a la enfermería de la época) que eran los encargados de ayudar (Arredondo, 2013). Como ejemplo, en el Hospital Royal de Haslar, se podían ver en 1789 carteles colgados como este: “ que no tiren por los desagües suciedad, huesos, o trapos, sino que se lleven a los lugares destinados al efecto.... "Las enfermeras deben obedecer las órdenes de la matrona en cuanto a cambiar la ropa de la cama y de los pacientes, la sabanas una vez cada 15 días, las camisas una vez cada cuatro días, gorros calzoncillos y calceti- 


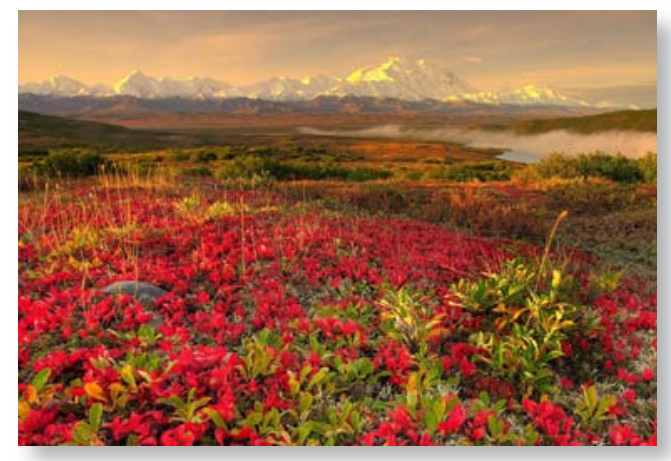

nes, una vez a la semana, o más a menudo si se resulta necesario".

La enfermería era más una ocupación religiosa que intelectual. Por tanto, el progreso científico se consideraba innecesario. Por otro lado, era muy difícil de convencer a personas validas que se dedicaran a la enfermería en los malolientes hospitales municipales, y en consecuencia, la enfermería retrocedió a su antigua posición de servil (Arredondo, 2013).

En general los asistentes o enfermeros laicos eran ignorantes, rudos y desconsiderados. Cuando una mujer no podía ganarse la vida con el juego o el vicio le quedaba la alternativa de convertirse en Enfermera. A esto hay que sumarlo, que el sueldo era escaso y a menudo lo completaban con todo tipo de sobornos ( Gallent, 1996).

En conclusión, la enfermería apenar estaba organizada y por supuesto carecía de posición social, llegando incluso las hermanas de las órdenes religiosas a estancarse por las restricciones económicas (Arratia, 2005).

Posteriormente, adentrándonos en el siglo XIX, se empezó a hacer latente el interés público para sacar de la situación en la que se encontraba la enfermería. Así, los médicos del clero y filántropos defendieron que la enfermería debería estar bajo el auspicio religioso o inmersas en un plan seglar con remuneración. Esta situación, dio lugar a la reforma estable de la enfermería (Chaverri, 1984).
En este siglo para las tareas de los cuidados a enfermos y pobres aparecen las Hermanas Católicas de la Merced, las Hermanas Protestantes de la Merced, las Hermanas de la Caridad, la Casa de San Juan, los Amigos de los Pobres y las Diaconisas de Kaisenwert. Se crean las primeras escuelas y aparecen los primeros textos escritos sobre la enfermería (Aróstegui,2011).

Esta reforma se extiende por todo el mundo y llega a España, donde destaca dentro de la Congregación de las "Las Hijas de la Caridad" de Concepción Arenal. Y es que, en España este lado oscuro de la enfermería que se vivió en Londres Francia, EEUU, no se vivió de forma tan patente dado que las órdenes religiosas siguieron atendiendo en los hospitales (Granjel, 1979).

El siglo XIX está marcado principalmente por la Revolución Industrial, que marcará un hito en los estilos de vida de la población, y en consecuencia, del proceso de enfermedad. Las enfermedades infecciosas como: la rabia, el paludismo, la tuberculosis, sífilis, tétanos, estuvieron muy presentes en el siglo XIX junto con otras enfermedades crónica como la diabetes y el reumatismo (Martinez, 2008).

De esta forma, a mediados del siglo XIX, los manuales se centraban en la descripción anatómica del cuerpo humano y en la exposición de intervenciones y acciones de cuidado, indicadas por el médico, según los síntomas que presentara la persona enferma, tales como: vómitos, estreñimiento, sangrado, diarreas (Arredondo, 2013).

Los cuidados generales que se proporcionaron en el siglo XIX dependían de los síntomas y de indicaciones médicas específicas. Ante una persona enferma, se debía de tener en cuenta la limpieza de su habitación y su ropa, el mantenimiento de la temperatura e 
iluminación, la alimentación, comodidad en la cama y la disponibilidad de objetos como orinales y escupideras. Para proporcionar estos cuidados, se formaba a profesionales proporcionándoles conocimientos limitados sobre las enfermedades. De esta manera, en los manuales de los siglos XIX, se nombran algunas enfermedades pero no se describe la patogenia. Por lo tanto, se cuidaba a todos los enfermos de la misma manera (Martínez, 2008)

Posteriormente, el rápido desarrollo de las ciencias aplicadas dio lugar a la aparición de técnicas de diagnóstico y tratamiento de las enfermedades, ofreciendo una prestación de servicios más amplia que contribuyó al desarrollo de la actividad médica curativa y administrativa para mejorar la calidad de vida (Siles, 2004).

Con lo que corresponde al mundo material en referencia a los cuidados, es en este siglo, donde los hospitales comienzan a organizarse de forma diferente. Se comienza a aplicar los resultados de la investigación básica (especialmente en física y química) y se introduce de manera rudimentaria la tecnología en los hospitales para el diagnóstico de enfermedades. Ejemplos de esa tecnología son: el termómetro, el pulsímetro para medir la frecuencia cardiaca y el fonendoscopio para auscultar ruidos cardiacos y pulmonares. Si bien, estos instrumentos irán evolucionando gradualmente. Además, como consecuencia de la investigación química, se comienzan a realizar análisis de la orina y de elementos básicos como glucosa (Siles, 2004).

En los manuales y la bibliografía consultada están presentes cuatro figuras que dedican su labor a cuidar: sangradores, enfermeras, practicantes y matronas.

La figura de los sangradores fue crucial durante el siglo XIX, sin embargo despareció a fi- nal de siglo, siendo relevada su labor por practicantes y posteriormente por enfermeras. Los sangradores se dedicaban de forma exclusiva a realizar sangrías, curaciones y algunas veces extracciones dentarias. Para ello, utilizaban diversos materiales entre los que se encuentran las lancetas o sanguijuelas. El cumplimiento de sus funciones exigía ciertos requisitos no solo teóricos, sino también en promoción de la higiene $\left(. . .5^{\circ}\right.$ Debe ser pródigo en el número de lancetas teniéndolas siempre limpias y bien vaciadas, puesto esto dará seguridad y facilidad en la operación...). En la bibliografía consultada, también se aprecia como las practicantes y enfermeras llevan objetos de la vida cotidiana al escenario donde daban lugar los cuidados. Por ejemplo, un plato que era utilizado para comer, se convertía en una bandeja para instrumentos, un pañuelo podía ser utilizado como venda y una camiseta como cabestrillo (Arratia, 2005).

También, era común suplir un instrumento necesario para los cuidados. Normalmente, se utilizaban objetos que provenían de otro contexto diferente al de cuidado y se les daba un nuevo uso. Por ejemplo, en la sangría la vasija para sangrar podía ser reemplazada por un acopa graduada de laboratorio o por un recipiente de la cocina y las ventosas por un vaso común (Aróstegui, 2001).

Es en este siglo donde adquiere gran significado histórico el Libro: "El Arte de la Enfermería", escrito por los Hermanos de la Orden de San Juan de Dios en el año 1833, y cuyo fin fue instruir a los enfermeros de esta Orden sobre el cuidado a los enfermos8. Con los reformadores religiosos y seglares se inicia la profesionalización de la actividad de cuidar, destacan: los Fliedner, Florence Nightingale y las órdenes surgidas en la Edad Moderna.

También hay que destacar la labor de Las 
diaconisas de Kaiserswerth, las cuales fueron preparadas por los Fliedner con una organización y un sistema de aprendizaje innovador que fue copiado posteriormente por otros países y ha mantenido en parte en la formación de las actuales enfermeras europeas. Destacaron también Elizabeth Fry, fundadora del instituto de hermanas de enfermeras protestantes, el Instituto Bishopgate, considero la calidad de la enfermería no por el ejercicio de la actividad como vocación religiosa (Chaverri, 1984).

La evolución de los cuidados, hizo que en el siglo XX las practicas que se llevaban a cabo estuviesen guiadas por condiciones de asepsia, antisepsia y vacunación.

\begin{tabular}{|c|c|}
\hline Aguja bifurcada. & $\begin{array}{l}\text { 1940.La aguja bifurcada ( con dos puntas) se } \\
\text { utilizaba para inmunizar de la viruela. }\end{array}$ \\
\hline Aguja de un $\mathrm{s} \square$ lo uso & $\begin{array}{l}\text { Su uso empez } \square \text { alrededor de } 1960-1970 \text {. Una } \\
\text { vez empleadas se desechaban pero las jeringas } \\
\text { se volvan a utilizar. }\end{array}$ \\
\hline Aguja de platino & $\begin{array}{l}\text { 1960.M }\lceil\mathrm{s} \text { costosas que las agujas reutilizables. } \\
\text { Se reconoc an por tener el ca } \square \mathrm{n} \text { dorado. }\end{array}$ \\
\hline Jeringa Barthelemy & $\begin{array}{l}\text { 1940. Jeringa de vidrio y cono tipo Luer. Se } \\
\text { fabric } \square \text { para soluciones de aceite gris (remedio } \\
\text { utilizado para el tratamiento mercurial de la } \\
\text { s filis) }\end{array}$ \\
\hline $\begin{array}{l}\text { Jeringa de Transfussi } \sqsubset \mathrm{n} \\
\text { Dott Jube }\end{array}$ & $\begin{array}{l}\text { 1940.Jeringa de doble va (aspirante-impelente). } \\
\text { Se utilizaba en transfusiones directas de persona } \\
\text { a persona. Su uso requer a cierta rapidez porque } \\
\text { no se utilizaba anticoagulante alguno. }\end{array}$ \\
\hline Jeringa Record 20cc-50cc & $\begin{array}{l}\text { 1960. Se requer a de un dispositivo adicional } \\
\text { conocido como record o alargadera para } \\
\text { utilizarse con agujas europeas. La esterilizaci } \square \text { n } \\
\text { se realizaba por ebullici } \square \text { n continua o } \\
\text { tindalizaci } \llbracket \text { n. }\end{array}$ \\
\hline Jeringa tipo Luer & $\begin{array}{l}\text { 1980.Jeringa de cristal con cono c } \square \text { ntrico } \\
\text { met } \square \text { lico en gris. Se utilizaba para va parenteral } \\
\text { y en la extracci } \llbracket \text { n de sangre. }\end{array}$ \\
\hline Jeringa tipo Luer de $1 \mathrm{cc}$ & $\begin{array}{l}\text { 1980. El tono azul del } \llbracket \text { mbolo esmerilado } \\
\text { facilitaba visualizar con mayor precisi }\lceil\text { n el } \\
\text { volumen de 1 quido a administrar. }\end{array}$ \\
\hline
\end{tabular}

Así, la formación de practicantes se fue ampliando y se fueron proporcionando cuidados más específicos aunque sujetos a prescripción médica. Por ello, a medida que se reconoce efecto beneficioso para la salud, la habitación y la cama adquieren fuerza en el contexto material de la salud. Por ello, se empieza a reclamar un ambiente óptimo para el enfermo con unas condiciones de construcción adecuadas. Así, el tamaño y la altura de las ventanas, el mobiliario, ventilación, las dimensiones de la habitación y orientación al sol eran factores que se tenían en cuenta a la hora de construcción de habitaciones en los hospitales sobre todo a mediados del siglo XX. Lo mismo ocurría con los domicilios, donde se cuidaba a la persona cuando enfermaba, y se tenían en cuenta las condiciones físicas y ambientales de la habitación, formando una parte central de la organización de los cuidados generales (León, 2008).

Con lo que respecta al material utilizado, la principal característica es que eran reutilizables. Como por ejemplo: las jeringas de cristal, los escarificadores, los guantes, los irrigadores, las sondas uretrales y las vendas. En la siguiente tabla se puede ver una breve explicación de las agujas y jeringas que se utilizaron a lo largo del siglo XX.

En los manuales y bibliografía consultada, no expresa el número de reutilizaciones que limitara la vida útil de un objeto. A veces incluso da la sensación de que la reutilización era indicación de mejor calidad del objeto (Arredondo, 2013).

Además, otra característica importante es la polivalencia de 
los objetos. Por ejemplo, la lámpara de alcohol para iluminar una habitación podía usarse para desinfectar objetos o para calentar un tubo de ensayo con orina en búsqueda de albúmina. Así, la polivalencia de los objetos permitía responder a la carencia de instrumentos específicos que estaban ausentes durante la realización del procedimiento.

Otro ejemplo de este hecho, se produce cuando las enfermeras utilizaban las agujas bifurcadas para inmunizar contra la viruela. A una de ellas se le ocurrió que para proteger de ese proceso de infecciones transformar un pequeño colador de metal en un protector para el brazo. Se hacía una perforación en el colador en dos puntos y se colocaba un lazo para poder sujetárselo al paciente, con el fin de permitir la ventilación y a su vez proteger del rascado que podía dar lugar como consecuencia del prurito19,20.

Posteriormente, los cambios acontecidos a lo largo del siglo XX, caracterizados por la conciencia social e importantes descubrimientos en diversos campos tales como la genética, la clonación de seres humanos, han supuesto un cambio en la concepción material de los cuidados de enfermería.

Así, los movimientos tecnológicos, científicos y sociales del siglo XX, han tenido un efecto significativo sobre el desarrollo de los cuidados de salud y sobre las políticas de los servicios de enfermería (Arratia, 2005).

\section{DISCUSIÓN Y CONCLUSIONES}

Son diversos los historiadores que se han centrado en señalar la transición de los cuidados en la historia desde su orígenes hasta la actualidad (Hernandez, 1995).

Particularmente, en Norteamérica existe una gran tradición de estudios sobre la historia de enfermería e incluso sobre los medios que se utilizan en la práctica enfermera. Entre los diversos trabajos, cabe destacar el de Fairman y Lynaugh en el que se expone el impacto del uso de la tecnología en la labor de enfermería (Fairman, 1998).

Por otro lado, Sandelowsky da visibilidad a los cuidados de enfermería mediante la explicación del uso de los objetos (Sandelowsky, 2000). También Zulumas recoge testimonios orales de enfermeras que trabajan en las unidades de cuidados críticos durante la década de los 50 para dilucidar el cambio tecnológico que ha tenido lugar a lo largo de este tiempo en estas unidades, así como el cambio de rol y las nuevas habilidades demandadas (Zulumas, 1995).

La necesidad de estudiar el campo que representa la tecnología empleada en los cuidados de enfermería, fue expuesto por Colliere en la década de los 80 del siglo pasado. Colliere argumentaba que la tecnología siempre ha estado presente en la labor del cuidado por eso es importante conocer su función (Siles, 2011).

El análisis de los medios y objetos utilizados en el cuidado, permite trazar una visión general acerca de la evolución del rol de los profesionales de enfermería (Siles,2000).

Existe una notable tradición a la labor enfermera en el siglo XVI, pero es en la época de la Ilustración, donde enfermería recuperaría una parte del terreno perdido en la centuria anterior, llegando hasta la actualidad. Así, el mundo material de los cuidados se ha construido y enriquecido gracias a las diferentes figuras que se han dedicado a cuidar y a los objetos que han formado parte de ese cuidado. Por esto, es importante recalcar el legado histórico de los materiales utilizados para proporcionar cuidados donde queda como evidencia los cuidados y los cuidadores (Siles, 2010). 


\section{FUENTES}

- Calvoy Martin, Jose. Normaluso del practicante. 1866. Disponible en: http://www.codem.es/Adjuntos/CODEM/ Documentos/Informaciones/Publico/04e2d096-e84e49aa-9ee5-e8a8dbbcd192/B58DCFE4-FA8A-4304A4BC-638D0EAF4611/83c67db7-5b41-4ee5-9f907e986bd28446/1866\%20Manual\%20para\% 20el\%20 uso\%20de\%20practicantes.pdf

- Fernándes-Corredor Mariano, Chicote. Manual de la enseñanza enfermeras. 1917.Disponible en: http:// www.codem.es/Adjuntos/CODEM/Documentos/ Informacion es/Publico/04e2d096-e84e-49aa-9ee5e8a8dbbcd 192/FDA4C5FA-4CA1-4E62-AE2F12FC19914C82/1dbb9f04-8aab-4b08-89e0$908 \mathrm{f} 56447 \mathrm{ac} 3 / 1917 \% 20 \mathrm{manual} \% 20 \mathrm{de} \% 2$ 0enfermeras\%20-\%2050.pdf

- García Velázquez Gerónimo. Manual del practicante y del interno del hospital. 1901. Disponible en: http:// www.codem.es/Adjuntos/CODEM/Documentos/ Informacione s/Publico/04e2d096-e84e-49aa-9ee5e8a8dbbcd192/353E240C-B260-49E9-815E-096F0B2CDE2C/8 7e8ef5b-0000-4b13-ae18-413dea8d985a/19 01\%20manual\%2 0del\%20practicante $\% 20 \% 2018$.pdf

- González del Valle Ambrosio. Manual de flebotomianos ó sangradores. 1846. Disponible en: http://www.codem.es/Adjuntos/CODEM/Documentos/Informaciones/ Publico/04e2d096-e84e49aa-9ee5-e8a8dbbcd192/94995293-5A75-483B8254-A27F399D7084/17308406-ad7d-4694-8ceb $133105462 \mathrm{f} 02 / 1846 \% 20 \mathrm{manual} \% 20 \mathrm{de} \%$ 20flebotomianos\%20o\%20sangradores.pdf

- Solano Javier. Manual de la Enfermera. 1936. 71 páginas. Disponible en: http://www.codem.es/Adjuntos/CODEM/ Documentos/Informaciones/Publico/04e2d096-e84e49aa-9ee5-e8a8dbbcd192/c68a41d2-1673-480c-856dcaa8d671e181/cdc37fdb-7030-4f93-8a02-80fa732f74a9/ manual de la Enferme.pdf

- Museo Histórico de Enfermería [Internet]. Fundación Jose Llopis Diez. Disponible en: http://www.museohistoricodeenfermeria.org/index.php

\section{BIBLIOGRAFIA}

- Arratia Figueroa A. (2005). Investigación y documentación histórica en enfermería. Texto y Contexto Enferm, 14(4), 567-574.

- Arredondo González, C.P., y Siles González, J. (2005). Tecnología y Humanización de los Cuidados. Una mirada desde la Teoría de las Relaciones Interpersonales. Index de Enfermería, 2009; 18(1),32-36.

- Arredondo- González, C.P., De la Cuesta Benjumea C., Ávila Olivares J.A.(2013). El mundo material para los cuidados de enfermería. Index Enferm (Gran).22(12),65-69.

- Arredondo-González, C.P., De la Cuesta-Benjumea. C., Ávila-Olivares, J.A. (2015). Un mundo en transición. Objetos para los cuidados en España entre 1855 y 1955. Aquichan 15(3), 426-439.
- Aróstegui J. (2001).La investigación histórica: teoría y método. Barcelona: Crítica.

- Ávila Olivares, J.A. (2010). ¿Existió realmente una titulación oficial con el nombre de Ministrante? Cultura de los cuidados, 27, 12-29.

- Campos, R., Montiel, L.,\& Huertas, R. (2007).Antecedentes y breves apuntes históricos. Medicina, ideología e historia en España (siglos XVI-XXI). Madrid: Consejo Superior de Investigaciones Científicas (CSIC).

- Bernabeu Mestre, J. (1989). La actualidad historiográfica de la Historia Social de la Enfermedad. Revista de Demografía Histórica, 7(3), 23-36.

- Eseverri Chaverri, C. (1984).Historia de la enfermería española e hispanoamericana. Barcelona: Salvat.

- Fairman, J.,\& Lynaugh, J. (1998). Critical care nursing a history. Philadelphia: University of Pennsyvania Press.

- Gallent Marco, M. (1996). Historia e historias de los hospitales. Revista d' Historia Medieval, 7: 179-91

- García García, I., \& Gozalbes Cravioto, E. (2013).Surgimiento y desarrollo de la Historia de la Enfermería en España. Enferm. Glob, 12(30), 305-314. Disponible en http://scielo.isciii.es/scielo.php?script=sci_ arttext\&pid=S1695-61412013000200015\&lng=es.

- Granjel, L.S. (1979).La medicina española del siglo XVIII. Salamanca: Universidad.

- Hernandez Conesa, J.M. (1995). Historia de la enfermería: un análisis histórico de los cuidados de enfemería. Madrid: McGraw-Hill Interamericana.

- Herrera Rodríguez, F. (2003). Nota sobre la Historia de la Enfermería en España (1977- 2002). Llull. Boletín de Sociedad Española de Historia de la Ciencia,26, 157- 73.

- Iglesias, C. (2009). No siempre lo peor es cierto. Estudios sobre Historia de España. Madrid: Galaxia Gutemberg; 2009.

- León Sanz, P. (2008). Hacia una historiografía de la enfermedad: Prácticas, profesiones y enfermos. Memoria y Civilización, 11, 243-70.

- Martínez Martín, M.L.,\& Chamorro Rebollo,E. (2011). Historia de la Enfermería. Evolución histórica del cuidado enfermero. Barcelona: Elsevier.

- Martínez Ruiz, E., Pazzis Pi, E. (Eds.) (2008).Ilustración, ciencia y técnica en el siglo XVIII español. Valencia: Universidad.

- Sandelowski, M.(2000). Devises and desires: Gender,Technology and American Nursing. Chapel Hill and London: The university of North Carolina Press.

- Santo-Tomás,M. (1994).Historia de la Enfermería. En: C. Fernández Ferrín, M. Garrido Abejar y M. Santo-Tomás (Dirs.). Enfermería Fundamental. Barcelona: Masson, p. 3-131.

- Siles-González, J. (2000).Antropología, historia y enfermería.Cultura de los cuidados, 7, 5-7.

- Siles-González, J. (2004). La construcción social de la Historia de la Enfermería. Index de enfermería, 13(47, 07-10. 


\section{Cultura de las Cuidados}

- Siles Gonzalez, J, Solano-Ruiz, M.C., Fernández de Freitas, G., \& Oguisso T. (2010).Political Systems and the Perspective on Nursing Education during the Spanish Transition (from the Franco Regime to Democracy). A Contribution based on Nursing History. Aquichan, 10(3),192-203.
- Siles-González, J. (2011). Historia de la Enfermería en España. Madrid: Difusión y Avances en Enfermería.

- Sosa Velasco, A.J. (2010). Médicos escritores en España, 1855-1955. Nueva York: Tamesiswoodbrige.

- Zulumas, J. (1995). Caring in Crisis. An oral History of critical care Nursing. Philadelphia: University of Pennsyvania Press.

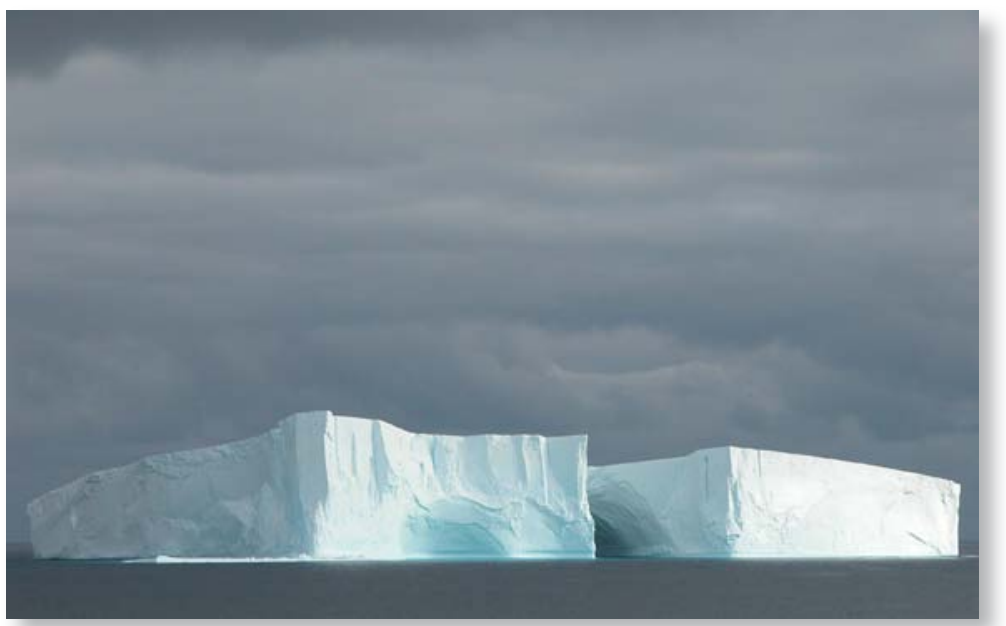

